\title{
Variable VHE gamma-ray emission from non-blazar AGNs
}

\author{
F. M. Rieger ${ }^{1,2}$ and F. A. Aharonian ${ }^{1,3}$ \\ 1 Max-Planck-Institut für Kernphysik, Saupfercheckweg 1, 69117 Heidelberg, Germany \\ e-mail: frank.rieger@mpi-hd.mpg.de \\ 2 European Associated Laboratory for Gamma-Ray Astronomy, jointly supported by CNRS and MPG \\ 3 Dublin Institute for Advanced Studies, 31 Fitzwilliam Place, Dublin 4, Ireland \\ Received 19 September 2007 / Accepted 6 December 2007
}

\section{ABSTRACT}

\begin{abstract}
Context. The observation of rapidly variable very high energy (VHE) gamma-rays from non-aligned active galactic nuclei (AGNs), as reported from M 87, proves challenging for conventional theoretical acceleration and emission models.

Aims. Motivated by recent work on pulsar-type particle acceleration in M 87 (Neronov \& Aharonian 2007, ApJ, 671, 85), we reexamine the centrifugal acceleration of particles by rotating jet magnetospheres in the vicinity of accreting supermassive black hole systems and analyze the energy constraints imposed for highly underluminous systems.

Methods. The maximum Lorentz factor for centrifugally accelerated electrons in the presence of inverse Compton losses, and the associated characteristic variability time scale, are determined. Applications are presented for conditions expected to be present in the radio galaxy $\mathrm{M} 87$, assuming accretion onto the central black hole to occur in an advection-dominated (ADAF) mode.

Results. We show that for a highly underluminous source like M 87, centrifugally accelerated electrons may reach Lorentz factors up to $\gamma \sim\left(10^{7}-10^{8}\right)$, allowing inverse Compton (Thomson) upscattering of sub-mm disk photons to the TeV regime. Upscattering of Comptonized disk photons results in a flat TeV spectrum $L_{v} \propto v^{-\alpha_{\mathrm{c}}}$ with spectral index $\alpha_{\mathrm{c}} \simeq 1.2$. The characteristic variability time scale is of the order $r_{\mathrm{L}} / c$, which in the case of M 87 corresponds to $\simeq 1.7 \mathrm{~d}$ for a typical light cylinder radius of $r_{\mathrm{L}} \simeq 5 r_{\mathrm{s}}$.

Conclusions. Centrifugal acceleration could provide a natural explanation for the challenging VHE emission features in M 87. Our results suggest that some advection-dominated accreting (non-blazar) AGNs could well be observable VHE emitting sources.
\end{abstract}

Key words. galaxies: active - galaxies: jets - radiation mechanism: non-thermal - gamma rays: theory - galaxies: individual: M 87

\section{Introduction}

The rapidly varying VHE gamma-ray flux, on time scales of days or less, observed in several AGNs implies a very compact VHE emission region of at most $R \leq c \Delta t \delta=2.6 \times$ $10^{15}(\Delta t / 1 \mathrm{~d}) \delta \mathrm{cm}$, where $\delta$ is the bulk Doppler factor of the VHE emitting region. For blazar sources with their jets pointing almost directly towards us (i.e., $\delta \sim \Gamma_{\mathrm{b}} \sim 15$ ), VHE variability on time scales of several hours may, in principle, be successfully accounted for by internal shock acceleration of electrons and inverse Compton upscattering of soft photons (e.g., Mastichiadis \& Kirk 2002). Yet, the fastest observed VHE blazar variations, on time scales of minutes (Gaidos et al. 1996; Aharonian et al. 2007; Albert et al. 2007), are generally difficult to understand within standard jet VHE emission models and are likely to require non-standard geometrical set-ups for their explanations (e.g., Salvati et al. 1998; Rieger 2004). In the case of non-aligned AGNs with $\delta \sim 1$, little can be gained from jet boosting, and it remains to be shown whether conventional, single homogeneous SSC models may be flexible enough to reproduce the VHE characteristics, including variability on time scales $\Delta t \lesssim 2$ day, as observed in the radio galaxy M 87 (Aharonian et al. 2006). Here we explore the possibility that centrifugal acceleration of electrons, occurring in the vicinity of a sub-Eddington accreting black hole system, could via inverse Compton processes lead to variable VHE gamma-ray emission. Centrifugal acceleration of plasma flows by rotating magnetospheres has been widely discussed, both in the context of pulsar emission models (e.g., Gold 1969; Machabeli \& Rogava 1994; Chedia et al. 1996; Gangadhara 1996; Bogovalov 1997;
Contopolous et al. 1999; Machabeli et al. 2005; Thomas \& Gangadhara 2007) and in the context of relativistic jet formation (e.g., Blandford \& Payne 1982; Fendt 1997; Camenzind 1999; Meier et al. 2001). Detailed MHD simulations, for example, indicate that centrifugally-driven outflows from AGNs could reach bulk Lorentz factors $\Gamma_{\mathrm{b}} \sim 10$. It has been realized for almost a decade that the generated MHD jet topology could also allow efficient centrifugal acceleration of relativistic charged test particles to very high energies in sub-Eddington accreting black hole systems (Gangadhara \& Lesch 1997; Rieger \& Mannheim 2000, henceforth RM00; Xu 2002; Osmanov et al. 2007). Here we examine this issue in more depth for parameters relevant to the radio galaxy M 87.

\section{Centrifugal acceleration of test particles}

\subsection{Particle energization and acceleration efficiency}

We consider an idealized two-dimensional model topology where the magnetic field is assumed to rotate rigidly with a fraction of the rotational velocity of the black hole (e.g., Fendt 1997), and where the electric field component parallel to the magnetic field line is screened by the magnetospheric jet plasma. A charged test particle, injected at the base and corotating with the field line (bead-on-wire motion), will then experience the centrifugal force and gain rotational energy while moving outward along the field (Machabeli \& Rogava 1994; Machabeli et al. 1996; Gangadhara \& Lesch 1997; RM00). The radial particle motion can be most conveniently analyzed in the framework of Hamiltonian dynamics (RM00) by noting that the Hamiltonian $H$ for a particle (rest mass $m_{0}$ ) on a relativistically rotating wire is a constant of motion, and is given by 
$H=\gamma m_{0} c^{2}\left(1-\Omega^{2} r^{2} / c^{2}\right)$, where $\gamma=1 /\left(1-\Omega^{2} r^{2} / c^{2}-\dot{r}^{2} / c^{2}\right)^{1 / 2}$ is the Lorentz factor, $\Omega=c / r_{\mathrm{L}}$ the angular velocity of the field line and $r_{\mathrm{L}}$ the light cylinder radius. The resultant equation of motion

$\gamma \frac{\partial^{2} r}{\partial t^{2}}+\frac{\partial r}{\partial t} \frac{\partial \gamma}{\partial t}=\gamma \Omega^{2} r$

can be solved analytically yielding (Machabeli \& Rogava 1994; RM00)

$r(t)=r_{\mathrm{L}} \operatorname{cn}\left(\lambda_{0}-\Omega t\right)$

for the time-dependence of the radial coordinate $r$, assuming a particle to be injected at $r_{0}$ with Lorentz factor $\gamma_{0}$, where $\mathrm{cn}$ is the Jacobian elliptic cosine and $\lambda_{0}$ is a Legendre elliptic integral of the first kind, and

$\gamma(r(t))=\frac{1}{\sqrt{\tilde{m}}\left(1-\Omega^{2} r^{2}(t) / c^{2}\right)}$,

when expressed in terms of $r(t)$, with $\tilde{m}=1 /\left(\gamma_{0}\left[1-\Omega^{2} r_{0}^{2} / c^{2}\right]\right)^{2} \leq$ 1. If we neglect, for a moment, radiative losses and the breakdown of the bead-on-the-wire approximation, a particle would reach the light cylinder $r_{\mathrm{L}}$ within a time $t_{v}=\lambda_{0} / \Omega \leq r_{\mathrm{L}} / c$, where, as a consequence of the reversal of the centrifugal acceleration, it would change direction and move inward again (Machabeli \& Rogava 1994). Knowing the dependence of $\gamma$ on $r$, we can easily determine the local acceleration time scale (RM00)

$t_{\mathrm{acc}}=\frac{\gamma}{\dot{\gamma}}=\frac{c \sqrt{1-\Omega^{2} r^{2} / c^{2}}}{2 \Omega^{2} r \sqrt{1-\tilde{m}\left[1-\Omega^{2} r^{2} / c^{2}\right]}}$,

which for large $\gamma$ approaches $t_{\text {acc }}^{\text {asy }}=1 /\left(2 \Omega \tilde{m}^{1 / 4} \gamma^{1 / 2}\right) \simeq$ $16.6 \tilde{m}^{-1 / 4}\left(10^{6} / \gamma\right)^{1 / 2}\left(r_{\mathrm{L}} / 10^{15} \mathrm{~cm}\right)$ [s]. The (local) acceleration time scale thus decreases with increasing $\gamma$. Equation (3) implies that the particle Lorentz factor increases dramatically as a particle approaches the light cylinder. Therefore, in many cases the characteristic linear size of our region of interest is much smaller than the light cylinder and the curvature radius of the field, a fact that may qualify the presumed straight field line approach.

\subsection{Efficiency constraints and maximum particle energy}

In reality, unlimited growth will be prohibited by radiative energy losses, the breakdown of the bead-on-the-wire approximation or the bending of the field line with increasing inertia (Gangadhara \& Lesch 1997; RM00). Inverse Compton upscattering of accretion disk photons, for example, leads to an energy loss of the particle characterized by a time scale $t_{\text {cool }} \propto 1 / \gamma$ that decreases faster than $t_{\text {acc }}$ and so introduces a natural limitation. Indeed, only for highly underluminous AGN sources will centrifugal acceleration be sufficiently efficient to accelerate electrons well beyond Lorentz factors of one hundred (RM00; $\mathrm{Xu}$ 2002). Assuming the inverse Compton scattering process to be approximately describable by the Thomson limit for a quasiisotropic photon distribution with energy density $U_{\mathrm{ph}}\left[\mathrm{erg} / \mathrm{cm}^{3}\right]$, we have $t_{\text {cool }} \simeq 3 \times 10^{7} /\left[\gamma U_{\mathrm{ph}}\right] \mathrm{s}$. Balancing acceleration by cooling $\left(t_{\mathrm{acc}}=t_{\mathrm{cool}}\right)$ for electrons thus gives a maximum electron Lorentz factor (see also Osmanov et al. 2007)

$\gamma_{\max }^{\mathrm{IC}} \simeq 3.2 \times 10^{6} \frac{\sqrt{\tilde{m}}}{U_{\mathrm{ph}}^{2}}\left(\frac{10^{15} \mathrm{~cm}}{r_{\mathrm{L}}}\right)^{2}$.

Provided that the corotation condition can be satisfied for such a range of Lorentz factors. The latter qualification seems important, as it may well happen that the particle motion becomes so perturbed by radiation recoil that the bead-on-the-wire approximation is no longer a useful concept. For highly underluminous AGN sources with, e.g. $U_{\mathrm{ph}} \lesssim 0.01 \mathrm{erg} / \mathrm{cm}^{3}$ and $r_{\mathrm{L}} \simeq 5 \times 10^{15} \mathrm{~cm}$ (see below, Sect. 3), for which Eq. (5) would otherwise imply $\gamma_{\max }^{\mathrm{IC}} \gtrsim 1.3 \times 10^{9} \tilde{m}^{1 / 2}$, this may in fact be the case. Indeed, from the bead-on-wire requirement that the radiation reaction force that results from inverse Compton scattering, i.e., $F_{\text {rad }} \simeq P_{\mathrm{IC}} / c$ (with $P_{\text {IC }}$ the single particle Thomson power) should be (much) smaller than the Lorentz force $F_{\mathrm{L}}$, one finds that achievable electron Lorentz factors should be (much) smaller than

$\gamma_{\max }^{\mathrm{RR}} \simeq 7.3 \times 10^{8}\left(\frac{B\left(r_{\mathrm{L}}\right)}{10 \mathrm{G}}\right)^{1 / 2}\left(\frac{0.01}{U_{\mathrm{ph}}}\right)^{1 / 2}$,

where $B\left(r_{\mathrm{L}}\right)$ is the magnetic field strength at the light cylinder radius. Yet, even for cases where radiative losses might be neglected, the breakdown of the bead-on-the-wire approximation (roughly occurring when the Coriolis force exceeds the Lorentz force) will prevent a particle from achieving infinite energies (RM00). In the simplest case, this restricts achievable particle energies to Lorentz factors below ${ }^{1}$

$\gamma_{\max }^{\mathrm{BB}} \simeq 2.0 \times 10^{8} \tilde{m}^{-1 / 6}\left(\frac{B\left(r_{\mathrm{L}}\right)}{10 \mathrm{G}}\right)^{2 / 3}\left(\frac{m_{\mathrm{e}}}{m_{0}}\right)^{2 / 3}\left(\frac{r_{\mathrm{L}}}{10^{15} \mathrm{~cm}}\right)^{2 / 3}$

which implies lower Lorentz factors for protons than for electrons. In any case, once the Lorentz factors become too high, the inertia of the particles overcomes the tension in the field line, so that the line is swept back opposite to the sense of rotation, slowing down acceleration and introducing curvature radiative losses, thus ultimately preventing infinite energy growth. In what follows, it is assumed that achievable Lorentz factors $\gamma$ always satisfy the relation $\gamma<\min \left\{\gamma_{\max }^{\mathrm{IC}}, \gamma_{\max }^{\mathrm{RR}}, \gamma_{\max }^{\mathrm{BB}}\right\}$.

\section{Application to the radio galaxy $M 87$}

\subsection{Phenomenological background}

The nearby (distance $\sim 16 \mathrm{Mpc}$ ) giant elliptical galaxy M 87 hosts one of the most massive black holes $M_{\mathrm{BH}} \simeq 3 \times 10^{9} M_{\odot}$ (e.g., Marconi et al. 1997), with Schwarzschild radius $r_{\mathrm{s}}=$ $2 G M_{\mathrm{BH}} / c^{2} \simeq 8.9 \times 10^{14} \mathrm{~cm}$, and a prominent one-sided $(\mathrm{kpc}-$ scale) jet visible from radio to X-ray wavelengths (e.g., Marshall et al. 2002; see Ly et al. 2007, for a possible radio counter-jet detection). HST observations have revealed superluminal motion of jet components at $\sim 0.5 \mathrm{kpc}$ from the central black hole, indicative of bulk flow Lorentz factors $\Gamma_{\mathrm{b}} \sim 6$ and a jet orientation of $\theta \sim 19^{\circ}$ to the line of sight (Biretta et al. 1999; but see also Ly et al. 2007, for larger radio $\theta$ ), suggesting that M 87 is a non-blazar jet source, characterized by only moderate Doppler factors. Superluminal radio features have also been detected in HST-1 located at around 100 pc (Cheung et al. 2007), although no superluminal motion has been found on small scales (Kovalev et al. 2007). HESS observations have recently shown M 87 to be a rapidly variable (observed time scale of $\sim 2$ days) $\mathrm{TeV}$ emitting source, yet with a relatively low (isotropic) $\mathrm{TeV}$ luminosity of several times $10^{40} \mathrm{erg} / \mathrm{s}$ (Aharonian et al. 2006). The total nuclear (disk and jet) bolometric luminosity of M 87 has been estimated to be of order $L_{\text {bol }} \sim 10^{42} \mathrm{erg} / \mathrm{s}$ or less

\footnotetext{
1 We are grateful to Osmanov et al. (2007) for making us aware of an incorrect conclusion in RM00. Although formula (19) in RM00 is correct, an inconsistent set of parameters has been used to estimate $\gamma_{\max }^{\mathrm{BB}}$ for the applications presented. This becomes relevant for highly underluminous sources, where achievable Lorentz factors can be much higher than previously concluded in RM00.
} 
(Reynolds et al. 1996; Owen et al. 2000), indicating that M 87 is a highly underluminous source with $l_{\mathrm{e}} \leq 3 \times 10^{-6}$, where $l_{\mathrm{e}}=L_{\mathrm{bol}} / L_{\mathrm{Edd}}$ and $L_{\mathrm{Edd}}$ is the Eddington luminosity. This has led to the proposal that M 87 is a prototype galaxy, where accretion occurs in a two-temperature, advective-dominated (ADAF) mode characterized by an intrinsically low radiative efficiency (Reynolds et al. 1996; Camenzind 1999; Di Matteo et al. 2003). Magnetic flux dragged inwards may then build up a rigidly rotating, dipolar magnetosphere, along which disk plasma can be centrifugally accelerated to (bulk) outflow Lorentz factors of $\Gamma_{\mathrm{b}} \simeq(5-10)$. The generated light cylinder scale is likely to be of order $r_{\mathrm{L}} \sim 5 r_{\mathrm{s}}$ (Camenzind \& Krockenberger 1992; Fendt 1997; Camenzind 1999; cf. also Fendt \& Memola 2001, for higher $r_{\mathrm{L}}$ if differential rotation is important). The magnetic field lines in global (quasi force-free) MHD wind solutions are radial near the black hole horizon, but asymptotically collimated into a cylindrical structure beyond the light cylinder, typically on radial scales $\sim 10 r_{\mathrm{L}}$. This seems consistent with high frequency VLBI observations in M 87, indicating a jet that forms with opening angles $\gtrsim 60^{\circ}$ at the jet base (Ly et al. 2007), as expected in MHD models, and a jet radius of $\sim 50 r_{\mathrm{s}}$ close to the origin (Krichbaum et al. 2006). It has been proposed recently that efficient pulsartype particle acceleration may occur in such an environment (Neronov \& Aharonian 2007, henceforth NA07).

\subsection{Implications for particle acceleration}

Let us consider the implications of these findings for the centrifugal acceleration of particles in M 87:

(1) Firstly, even for the most limiting case where all of the observed bolometric luminosity is assumed to originate within a region $r_{\mathrm{L}}$, so that the energy density of the radiation field may be approximated by $U_{\mathrm{ph}}=L_{\mathrm{bol}} /\left(4 \pi r_{\mathrm{L}}^{2} c\right)$, Eq. (5) would imply that Lorentz factors $\gamma_{\max }^{\mathrm{IC}} \simeq 10^{7} \tilde{m}^{1 / 2}$ can be achieved, allowing Thomson upscattering of infrared $(\geq 0.01 \mathrm{eV})$ photons to the $\mathrm{TeV}$ regime. In reality, this case is certainly over-restrictive, as it assumes that neither the observed jet nor the disk regions beyond $r_{\mathrm{L}}$ make a significant contribution to the bolometric luminosity output, which we consider unlikely. Indeed, if the relevant luminosity is an order of magnitude or more smaller, as expected in the ADAF scenario (see estimate $L_{R}$ below), the maximum Lorentz factors implied by Eq. (5) will be at least two orders of magnitude higher. This suggests that electron Lorentz factors up to $\gamma \sim\left(10^{7}-10^{8}\right)$ may be well possible (cf. Eqs. (5), (6)).

(2) If accretion in M 87 indeed occurs in an ADAF mode, the emitted disk spectrum will range from the radio up to the $\mathrm{X}$-ray regime and beyond: the radio part is produced by synchrocyclotron emission of thermal electrons $\left(T_{\mathrm{e}} \simeq 5 \times 10^{9} \mathrm{~K}\right)$, the optical/UV/X-rays arise via inverse Compton scattering of radio soft photons, and the hard X-rays are due to bremsstrahlung and multiple Compton scattering (Mahadevan 1997; Narayan et al. 1998; Yi 1999). The ADAF equipartition magnetic field for M 87 is of order $B_{\text {eq }} \sim 2.5 \times 10^{4} \dot{m}^{1 / 2}\left(r / r_{\mathrm{s}}\right)^{-5 / 4} \mathrm{G}$, where $\dot{m}$ is the accretion rate in units of the Eddington rate (cf. Yi 1999). For the inferred Bondi accretion rate $\dot{m}=\dot{m}_{\mathrm{b}} \sim 1.6 \times 10^{-3}$ (Di Matteo et al. 2003) this gives $B\left(r_{\mathrm{s}}\right) \sim 10^{3} \mathrm{G}$, suggesting possible (radial) jet magnetic field strengths close to the light cylinder of $B\left(r_{\mathrm{L}}\right) \sim(10-50) \mathrm{G}$. The highest radio emission in an ADAF is generally produced in the innermost region of the accretion flow. For M 87, the characteristic synchrotron (peak) frequency becomes

$v_{\mathrm{s}}(r) \simeq 4 \times 10^{13} \dot{m}_{\mathrm{b}}^{1 / 2}\left(\frac{r}{r_{\mathrm{s}}}\right)^{-5 / 4}\left(\frac{T_{\mathrm{e}}}{5 \times 10^{9} \mathrm{~K}}\right)^{2} \mathrm{~Hz}$, and the associated radio luminosity $L_{R} \sim v_{\mathrm{s}} L_{v}^{\mathrm{s}}$ is given by

$L_{R} \simeq 10^{42} \dot{m}_{\mathrm{b}}^{4 / 5}\left(\frac{x_{\mathrm{M}}}{10^{3}}\right)^{8 / 5}\left(\frac{T_{\mathrm{e}}}{5 \times 10^{9} \mathrm{~K}}\right)^{21 / 5}\left(\frac{v}{10^{11} \mathrm{~Hz}}\right)^{7 / 5} \mathrm{erg} / \mathrm{s},(9)$

where $\left(x_{\mathrm{M}} / 10^{3}\right) \sim 1$ denotes the dimensionless synchrotron selfabsorption frequency (Yi \& Boughn 1998). On the light cylinder scale $\left(r_{\mathrm{L}} \simeq 5 r_{\mathrm{s}}\right)$ this implies a peak frequency $v_{\mathrm{s}}\left(r_{\mathrm{L}}\right) \simeq$ $2 \times 10^{11} \mathrm{~Hz}$ and a luminosity $L_{R} \sim 2 \times 10^{40} \mathrm{erg} / \mathrm{s}$. Thomson upscattering $\left(v \sim \gamma^{2} v_{\mathrm{s}}\right)$ of these mm soft photons by centrifugally accelerated electrons with $\gamma$ up to $\sim\left(10^{7}-10^{8}\right)$ will thus, in principle, lead to VHE photons with energies up to $\sim(0.1-10) \mathrm{TeV}$. Comptonization of cyclosynchrotron soft photons adds a power law tail to the disk spectrum above $v_{\mathrm{s}}$, i.e., $L_{v} \simeq L_{v_{\mathrm{s}}}\left(v / v_{\mathrm{s}}\right)^{-\alpha_{\mathrm{c}}}$ with power index $\alpha_{\mathrm{c}}=-\ln \tau_{\mathrm{es}} / \ln A$ (Mahadevan 1997). For M 87 with $\dot{m}_{\mathrm{b}}=1.6 \times 10^{-3}$, viscosity parameter $\alpha=0.3$ and $T_{\mathrm{e}}=5 \times 10^{9} \mathrm{~K}$, we obtain an electron scattering depth $\tau_{\mathrm{es}} \simeq 0.04$ and an amplification factor $A \simeq 15.7$, so that $\alpha_{\mathrm{c}} \simeq 1.2$.

(3) Suppose that during an active state, test particles are injected with $\gamma_{0} \gtrsim 2$ at a constant rate $Q$ and accelerated up to a threshold $\gamma_{\mathrm{b}}<\gamma_{\max }^{\mathrm{RR}}<\gamma_{\max }^{\mathrm{IC}}$, above which they are considered to leave the centrifugal acceleration process due to the breakdown of corotation. The differential particle energy distribution $n(\gamma)$ along a field line would satisfy the simplified transport equation

$$
\frac{\partial n}{\partial t}+\frac{\partial}{\partial \gamma}\left(\left[\frac{\gamma}{t_{\mathrm{acc}}}-\frac{\gamma}{t_{\mathrm{cool}}}\right] n\right)-\frac{n}{\tau_{\mathrm{esc}}} \delta\left(\gamma_{\mathrm{b}}-\gamma\right)=Q \delta\left(\gamma-\gamma_{0}\right)
$$

with $t_{\text {cool }} \propto \gamma^{-1}$ and $t_{\text {acc }} \propto \gamma^{-1 / 2}$ (cf. Eq. (4)). Above injection, the steady-state distribution in the acceleration region thus becomes

$n(\gamma) \propto\left(1-\sqrt{\frac{\gamma}{\gamma_{\max }^{\mathrm{IC}}}}\right)^{-1} \gamma^{-3 / 2} H\left(\gamma_{\mathrm{b}}-\gamma\right)$,

i.e., a power law distribution with index $-3 / 2$ for $\gamma_{\mathrm{b}} \ll \gamma_{\max }^{\mathrm{IC}}$. The emergent (singly scattered, Thomson) inverse Compton spectrum $j_{\mathrm{IC}}(v)$ from such a hard electron distribution would follow a power law $j_{\mathrm{IC}}(v) \propto v^{-\alpha}$ with index $\alpha=0.25$ for $v \ll 4 \gamma_{\mathrm{b}}^{2} v_{\mathrm{s}}$. Integrating Eq. (11) over $\gamma$ gives the number of particles along a field line $N \simeq Q / \Omega$. Electrons, escaping quasi-monoenergetically with $\gamma_{\mathrm{b}} \sim 10^{7}$ from the acceleration mechanism and encountering the Comptonized disk photons $\left(v>v_{\mathrm{s}}\right)$, can Thomson upscatter them to the $\mathrm{TeV}$ regime, producing a power law-like energy distribution above $4 \gamma_{\mathrm{b}}^{2} \nu_{\mathrm{s}}$ with index $\alpha_{\mathrm{c}} \sim 1.2$, consistent with the value $1.22 \pm 0.15$ derived for the HESS 2005 observations of M 87 (Aharonian et al. 2006).

(4) The number of escaping particles per unit time is $n_{\mathrm{e}} \sim$ $n\left(\gamma_{\mathrm{b}}\right) \gamma_{\mathrm{b}} / \tau_{\mathrm{esc}}$. Thus, within some time smaller than the cooling time $\left(\Delta t=\rho t_{\text {cool }}, \rho<1\right)$, we accumulate $N_{\mathrm{b}} \sim n_{\mathrm{e}} \Delta t$ particles that can IC upscatter Comptonized disk photons. We can roughly estimate the associated TeV luminosity from $L_{\mathrm{IC}} \sim N_{\mathrm{b}} P_{\mathrm{IC}}$, where $P_{\mathrm{IC}}=1.3 \sigma_{\mathrm{T}} c \gamma_{\mathrm{b}}^{2} U_{\mathrm{ph}}$ is the single particle Compton power per unit volume. This gives

$L_{\mathrm{IC}} \sim 10^{40} \rho\left(\frac{\gamma_{\mathrm{b}}}{5 \times 10^{7}}\right)^{2}\left(\frac{N}{10^{36}}\right)\left(\frac{5 r_{\mathrm{s}}}{r_{\mathrm{L}}}\right) \mathrm{erg} / \mathrm{s}$.

To achieve a Compton luminosity comparable to the observed TeV luminosity of $L_{\mathrm{TeV}} \simeq 3 \times 10^{40} \mathrm{erg} / \mathrm{s}$ (Aharonian et al. 2006), we thus need $N \sim 3 \times 10^{36} / \rho$ particles along field lines. Denoting the relevant acceleration volume by $\Delta V \sim \eta r_{\mathrm{L}}^{2} \Delta r$, with characteristic length scale $\Delta r=|\gamma /(\partial \gamma / \partial r)| \sim\left(\gamma_{0} / \gamma_{\mathrm{b}}\right) r_{\mathrm{L}}$ and $\eta<1$, the corresponding kinetic energy density $n\left(\gamma_{\mathrm{b}}\right) \gamma_{\mathrm{b}}^{2} m_{\mathrm{e}} c^{2} / \Delta V$ (for 
$\left.\rho \eta \gtrsim 10^{-5}\right)$ is still well below the energy density $B^{2} /(8 \pi)$ of the magnetic field, suggesting that the presumed (quasi forcefree) MHD field structure is still a valid approximation (cf. also Osmanov et al. 2007).

(5) In principle, TeV gamma-rays can be strongly attenuated due to photon-photon pair production in the background disk photon field. The narrow dependence of the cross-section $\sigma_{\gamma \gamma}$ on the product of photon energies implies that VHE photons of energy $E$ interact most efficiently with infrared background photons of energy $\epsilon_{\mathrm{IR}} \simeq(1 \mathrm{TeV} / E) \mathrm{eV}$. The optical depth $\tau$ for a $\gamma$ ray photon in a background field of infrared luminosity $L_{\mathrm{IR}}$ and size $R_{\mathrm{IR}}$ thus becomes (cf. NA07)

$$
\tau\left(E, R_{\mathrm{IR}}\right)=\frac{L_{\mathrm{IR}} \sigma_{\gamma \gamma}}{4 \pi R_{\mathrm{IR}} \epsilon_{\mathrm{IR}}} \simeq 0.2\left(\frac{L_{\mathrm{IR}}}{10^{41} \mathrm{erg} / \mathrm{s}}\right)\left(\frac{r_{\mathrm{L}}}{R_{\mathrm{IR}}}\right)\left(\frac{E}{1 \mathrm{TeV}}\right),
$$

indicating that due to its low bolometric luminosity M 87 could be well transparent to VHE gamma-rays, even if almost all of the observed infrared luminosity $L_{\mathrm{IR}} \simeq 10^{41} \mathrm{erg} / \mathrm{s}$ (Whysong \& Antonucci 2004) is (somewhat unrealistically) taken to be produced on a scale $R_{\mathrm{IR}} \sim r_{\mathrm{L}}$. Note that even if $\tau$ would become larger than one, $\gamma$-rays from the last transparent layer are still able to escape, so that the VHE flux would not simply decrease exponentially by $\exp (-\tau)$, but only by a factor of $\sim \tau$ (NA07).

(6) The number of electrons escaping quasimonoenergetically from the acceleration mechanism in the vicinity of the light cylinder is of order $N_{\mathrm{b}}$. Once these energetic particles encounter non-vanishing perpendicular and/or the turbulent plasma magnetic fields, they can produce synchrotron emission arising as $L_{v} \propto v^{1 / 3}$ below, and decaying exponentially above the peak frequency $v_{\text {syn }} \sim 50\left(\gamma_{\mathrm{b}} / 5 \times 10^{7}\right)^{2}(B \sin \alpha / 1 \mathrm{G}) \mathrm{MeV}$ with a total luminosity of order $L_{\text {syn }} \sim P_{\text {syn }} N_{\mathrm{b}} \sim 0.06 L_{\mathrm{IC}}(B \sin \alpha)^{2}$, where $P_{\text {syn }}$ is the single particle synchrotron power. In order to satisfy the restrictions imposed by the existing (yet non-contemporaneous) upper limit on the M 87 flux in the EGRET energy band above $100 \mathrm{MeV}$ (e.g., Reimer et al. 2003), the effectively encountered fields should be smaller than $\sim 1$ Gauss. This seems consistent with independent estimates suggesting a strength of the random field component close to the black hole of below one Gauss (NA07). The overall spectral energy distribution in M 87 is then likely to consist of a number of different contributions, involving also other leptonic (Georganopoulos et al. 2003; NA07) and perhaps even hadronic (Reimer et al. 2004) processes. If so, then no straighforward X-ray-TeV correlation might be expected.

(7) As shown above, accelerating particles up to the light cylinder typically takes a time $r_{\mathrm{L}} / c$, suggesting a characteristic variability time scale for $\mathrm{M} 87$ of $t_{v} \simeq \frac{r_{\mathrm{L}}}{c} \sim \frac{5 r_{\mathrm{s}}}{c} \simeq 1.7$ days, well consistent with the observed $\mathrm{TeV}$ time scale of $\Delta t \sim 2$ days, a fact that may further validate the assumptions of the presented model.

\section{Conclusions}

VHE radiation from low-luminous, non-blazar AGN jet sources like M 87 could provide an ideal test laboratory for the analysis of particle acceleration processes close to the supermassive black hole event horizon. In blazars with their relativistic jets pointing towards us, most of these traces are likely to be masked by strong relativistic beaming effects, while for luminous quasars internal absorption of gamma-rays becomes dominant. Based on a simple toy model we have shown that efficient centrifugal acceleration of electrons in the vicinity of the light cylinder could provide a natural explanation for variable (time scale of one day) VHE emission with a hard inverse Compton spectrum as observed in M 87. Our models fits well with other evidence for advection-dominated accretion in M 87 and may indeed be regarded as providing some further corroboration for the presence of such modes in highly underluminous AGNs.

As always, there are a number of subtleties whose impact on the presented results need to be explored in more details including general relativistic effects, anisotropic scattering modifications, quasi rigid rotation and plasma instabilities. The extent to which our conclusions might be affected may require fully relativistic modelling. Yet, given the demonstrated potential of centrifugal acceleration and our current understanding of relativistic jet formation, this may represent a program worth pursuing.

Acknowledgements. Discussion with and comments by John Kirk, Christian Fendt and Karl Mannheim are gratefully acknowledged.

\section{References}

Aharonian, F., Akhperjanian, A. G., Bazer-Bachi, A. R., et al. (HESS collaboration) 2006, Science, 314, 1424

Aharonian, F., Akhperjanian, A. G., Bazer-Bachi, A. R., et al. (HESS collaboration) 2007, ApJ, 664, L71

Albert, J., Aliu, E., Anderhub, H., et al. (MAGIC collaboration) 2007, ApJ, 669, 862

Biretta, J. A., Sparks, W. B., \& Macchetto, F. 1999, ApJ, 520, 621

Blandford, R. D., \& Payne, D. G. 1982, MNRAS, 199,883

Bogovalov, S. V. 1997, A\&A, 327, 662

Camenzind, M. 1999, in The radio galaxy Messier 87, ed. H.-J. Röser, \& K. Meisenheimer, LNP 530, 252

Camenzind, M., \& Krockenberger, M. 1992, A\&A, 255, 59

Chedia, O. V., Kahniashvili, T. A., Machabeli, G. Z., \& Nanobashvili, I. S. 1996, Ap\&SS, 239, 57

Cheung, C. C., Harris, D. E., \& Stawarz, L. 2007, ApJ, 663, L65

Contopoulos, I., Kazanas, D., \& Fendt, C. 1999, ApJ, 511, 351

Di Matteo, T., Allen, S. W., Fabian, A. C., Wilson, A. S., \& Young, A. J. 2003, ApJ, 582, 133

Fendt, C. 1997, A\&A, 319, 1025

Fendt, C., \& Memola, E. 2001, A\&A, 365, 631

Gaidos, J. A., Akerlof, C. W., Biller, S. D., et al. (Whipple collaboration) 1996, Nature, 383, 319

Gangadhara, R. T. 1996, A\&A, 314, 853

Gangadhara, R. T., \& Lesch, H. 1997, A\&A, 323, L45

Georganopoulos, M., Perlman, E. S., \& Kazanas, D. 2003, ApJ, 634, L33

Gold, T. 1969, Nature, 221, 25

Krichbaum, T. P., Graham, D. A., Bremer, M., et al. 2006, J. Phys. Conf. Ser., 54,328

Kovalev, Y. Y., Lister, M. L., Homan, D. C., Kellermann, K. I. 2007, ApJ, 668, L27

Ly, C., Walker, R. C., \& Junor, W. 2007, ApJ, 660, 200

Machabeli, G. Z., \& Rogava, A. D. 1994, Phys. Rev. A, 50, 98

Machabeli, G. Z., Nanobashvili, I. S., \& Rogava, A. D. 1996, Radiophysics and Quantum Electronics, 39, 26

Machabeli, G. Z., Osmanov, Z. N., \& Mahajan, S. M. 2005, PhPL, 12, 2901

Mahadevan, R. 1997, ApJ, 477, 585

Marconi, A., Axon, D. J., Macchetto, F. D., et al. 1997, MNRAS, 289, L21

Marshall, H. L., Miller, B. P., Davis, D. S., et al. 2002, ApJ, 564, 683

Mastichiadis, A., \& Kirk, J. G. 2002, PASA, 19, 138

Meier, D. L., Koide, S., \& Uchida, Y. 2001, Science, 291, 84

Narayan, R., Mahadevan, R., \& Quataert, E. 1998, in Theory of Black Hole Accretion Disks, ed. M. A. Abramowicz, et al. (Cambridge), 148

Neronov, A., \& Aharonian, F. A. 2007, ApJ, 671, 85 (NA07)

Osmanov, Z., Rogava, A., \& Bodo, G. 2007, A\&A, 470, 395

Owen, F. N., Eilek, J. A., \& Kassim, N. E. 2000, ApJ, 543, 611

Reimer, A., Protheroe, R. J., \& Donea, A.-C. 2004, A\&A, 419, 89

Reimer, O., Pohl, M., Sreekumar, P., \& Mattox, J. R. 2003, ApJ, 588, 155

Reynolds, C. S., di Matteo, T., Fabian, A. C., Hwang, U., Canizares, C. R. et al. 1996, MNRAS, 283, L111

Rieger, F. M. 2004, ApJ, 615, L5

Rieger, F. M., \& Mannheim, K. 2000, A\&A, 353, 473 (RM00)

Salvati, M., Spada, M., \& Pacini, F. 1998, ApJ, 495, L19

Thomas, R. M. C., \& Gangadhara, R. T. 2007, A\&A, 467, 911

Whysong, D., \& Antonucci, R. 2004, ApJ, 602, 116

Xu, Y. D. 2002, A\&A, 381, 357

Yi, I. 1999, in Astrophysical Disks, ed. J. A. Sellwood, \& J. Goodman, ASP Conf. Ser., 160, 279

Yi, I., \& Boughn, S. P. 1998, ApJ, 499, 198 